PROCEEDINGS OF THE

AMERICAN MATHEMATICAL SOCIETY

Volume 138, Number 1, January 2010, Pages 267-273

S 0002-9939(09)10064-3

Article electronically published on August 24, 2009

\title{
NEBENHÜLLE AND THE GLEASON PROBLEM
}

\author{
LINUS CARLSSON
}

(Communicated by Mei-Chi Shaw)

\begin{abstract}
This article concerns the Gleason property as a local phenomenon. We prove that there always exists an open set where the domain $D \Subset \mathbb{C}^{2}$ has the Gleason $\mathcal{B}$ property whenever the boundary of the Nebenhülle of $D$ coincides with a $C^{2}$ smooth part of the boundary $b D$; here $\mathcal{B}$ is either one of the Banach algebras, $H^{\infty}$ or $A$. As an easy consequence of this, we see that if the extremal boundary points are $C^{2}$-smooth, then $D$ has the Gleason $\mathcal{B}$ property close to those points. Also a $\bar{\partial}$-problem for locally supported forms is solved.
\end{abstract}

\section{INTRODUCTION AND NOTATIONS}

This article addresses some aspects of the Gleason problem. The problem was first studied by Andrew Gleason. In his article, Gle64, Gleason studied the Banach algebra $A(B(0,1))$ consisting of those holomorphic functions defined on the unit ball in $\mathbb{C}^{n}$ which can be continuously continued up to the boundary. He asked whether the algebra $A(B(0,1))$ was finitely generated. He showed that if this was the case, then the maximal ideal consisting of functions vanishing at the origin is generated by the coordinate functions.

The question whether these ideals in algebras of holomorphic functions are generated by the coordinate functions has been named the Gleason problem.

For the time being, let $\mathcal{B}(D)$ denote an algebra of holomorphic functions on an open set $D \subset \mathbb{C}^{n}$.

We say that a domain $D \subset \mathbb{C}^{n}$ has the Gleason $\mathcal{B}$ property at $p \in D$ if for every $f \in \mathcal{B}(D)$ such that $f(p)=0$ there exist functions $f_{1}, f_{2}, \ldots, f_{n} \in \mathcal{B}(D)$ such that

$$
f(z)=\sum\left(z_{j}-p_{j}\right) f_{j}(z)
$$

for all $z \in D$.

Many authors have addressed the Gleason problem. The first one to solve Gleason's original question was Leibenzon; the details can be found in Hen71. Leibenzon actually proved that the algebra $A(B(0,1))$ has the Gleason $A$ property at every point in the unit ball, $B(0,1)$.

When a domain $D \Subset \mathbb{C}^{n}$ has the Gleason $\mathcal{B}$ property at every point in $D$, we simply say that $D$ has the Gleason $\mathcal{B}$ property.

The Gleason $\mathcal{B}$ property has been studied for different algebras of holomorphic functions defined on different types of domains; this has been done in various ways,

Received by the editors December 9, 2008, and, in revised form, May 26, 2009.

2000 Mathematics Subject Classification. Primary 32A65, 32W05, 46J20.

Key words and phrases. Holomorphic functions, Banach algebras, Nebenhülle, $\bar{\partial}$-problems.

(C)2009 American Mathematical Society 267

Reverts to public domain 28 years from publication 
for example by explicit methods (see e.g. [Hen71, Jak84, [BF95] or [LW02]) and by $\bar{\partial}$-solutions (see e.g. Bea80, [FØ83], Ceg93 and [CCF07]). The usual obstacle is the properties (or rather the lack of properties) the domain in question possesses.

Some counterexamples (see e.g. OA87, and [BF91]) are known; in these examples it is shown that there is a subset of the domain where the domain does not have the Gleason $\mathcal{B}$ property.

In view of these results, we have studied the Gleason problem locally in rather general domains. In this paper we show that under mild assumptions on the boundary there will always exist points where the Gleason $\mathcal{B}$ property holds.

In the remainder of this article $D$ will be a bounded domain in $\mathbb{C}^{n}$. We will study $\mathcal{B}(D)$, which will denote one of the Banach algebras, $H^{\infty}(D)$ (the bounded holomorphic functions on $D$ ) or $A(D)$ (holomorphic functions on $D$ which can be continuously continued to the boundary of $D$ ).

The boundary of $D$ will be denoted $b D$, and the set of strictly pseudoconvex boundary points will be denoted $S(b D)$. A point in space will have the form $z=$ $\left(z_{1}, z_{2}, \ldots, z_{n}\right) \in \mathbb{C}^{n}$, and $B(p, r)$ is the open ball with center at $p$ and radius $r$. By $\mathcal{Z}_{g}$ we denote the zero set of the function $g$.

We now present the main result of this paper, Theorem 11, which we state here, but postpone the proof until section 4 .

Theorem 1. Let $D \subset \mathbb{C}^{2}$ be a bounded domain such that $b D \in C^{2}$. If $\mathcal{B} \in$ $\left\{H^{\infty}, A\right\}$, then there always exist points in $D$ where we can solve the Gleason $\mathcal{B}$ problem.

With this theorem it is possible to modify the counterexample in BF91 to show that there exists a domain in which there is an open subset where the domain does not have the Gleason $\mathcal{B}$ property but in another open subset of the domain the Gleason $\mathcal{B}$ property holds true; see Car08a.

The outline of the paper is the following: In section 2 we show that the Gleason $\mathcal{B}$ property holds true for points close to strictly pseudoconvex boundary points of a certain kind. In section 3 we solve a simple $\bar{\partial}$-problem for locally supported forms. This is finally used in section 4 to give a more general description of domains where we can prove a local Gleason $\mathcal{B}$ property.

\section{A local solution to the Gleason problem}

In this section the domain $D$ will be a bounded domain in $\mathbb{C}^{2}$. The interesting result of this section is the following, rather technical, proposition.

Proposition 1. Let $D \subset \mathbb{C}^{2}$ be a bounded domain. Let $\xi \in b D$ and assume that there exists a function $\kappa_{\xi} \in H(\widetilde{D})$ for some pseudoconvex domain $\widetilde{D} \ni D$ such that $\kappa_{\xi}$ is a peaking function for $A(D)$ at $\xi$. Furthermore assume that there is a relatively open neighborhood $V$ of $\xi$ in $b D$ such that $V$ is $C^{1}$ smooth and if

$$
\begin{gathered}
\lambda \in L_{(0,1)}^{\infty}(\bar{D}) \cap \operatorname{ker} \bar{\partial} \text { and } \\
\operatorname{supp}(\lambda) \cap b D \Subset V,
\end{gathered}
$$

then there exists a function $u \in C(\bar{D})$ such that $\bar{\partial} u=\lambda$. Then there is a neighborhood $O_{\xi}$ of $\xi$ such that $D$ has the Gleason $\mathcal{B}$ property at $p$ for all $p \in O_{\xi} \cap D$.

Remark 1. The peaking functions $\kappa_{\xi}$ mentioned above do exist for a big set of strictly pseudoconvex boundary points. In fact, we shall later see that all bounded domains in $\mathbb{C}^{n}$ with a $C^{2}$-smooth boundary have uncountably many such points. 
To prove this proposition we start with a lemma.

Lemma 1. Let $g_{1}(z)=1-\kappa_{\xi}(z)$, where $\kappa_{\xi}, D$ and $\widetilde{D}$ satisfy the hypothesis of Proposition 1. There exist an affine linear function $g_{2} \in H\left(\mathbb{C}^{n}\right)$ and a neighborhood $\omega \subset \widetilde{D}$ of $\xi$ such that

$$
\mathcal{Z}_{g_{1}} \cap \mathcal{Z}_{g_{2}} \cap \omega=\{\xi\} .
$$

Proof. First we make a complex affine change of coordinates (we denote this mapping as $\sigma$, and let $T f=f \circ \sigma^{-1}$ for all functions $f$ on $\left.D\right)$ which takes $\xi$ to $(0,0)$ and such that the outward unit normal at the origin is $(0, i)$. We call the translated domain $D_{T}$.

Let $D_{2}$ be the one dimensional slice $\left\{z_{2}:\left(0, z_{2}\right) \in D_{T}\right\}$. Then $\kappa_{\xi}$ peaks at the origin and is therefore non-constant, so $T g_{1}\left(0, z_{2}\right)$ has an isolated zero at $(0,0)$.

Especially, there exists a neighborhood $U$ of 0 such that $T g_{1}\left(0, z_{2}\right)=0$ only when $z_{2}=0$.

Defining $T g_{2}\left(z_{1}, z_{2}\right):=z_{1}$ gives that

$$
\mathcal{Z}_{T g_{1}} \cap \mathcal{Z}_{T g_{2}} \cap U=\{0\} .
$$

Let $g_{2}$ be the reversed complex linear change of coordinates of $T g_{2}$ and let $\omega$ be the preimage of $U$ under the same transformation intersected with $\widetilde{D}$. This completes the proof.

Now we prove Proposition 1. In the part of the proof where the Gleason property is shown we follow the ideas in Bea80.

In the following we let $\nu_{\xi}$ denote the outward unit normal vector at $\xi$. Also, for a set $D \subset \mathbb{C}^{2}$ and a constant $r$, we define $D_{r}$ as

$$
D_{r}=\left\{z \in \mathbb{C}^{2}: z+r \nu_{\xi} \in D\right\} .
$$

Proof of Proposition 1. Let $g_{1}, g_{2}$ and $\omega$ be as in Lemma 1 .

We want the intersection of the zero sets of $g_{1}$ and $g_{2}$ to be inside the domain, so we translate the zero sets of the functions. This is done by an affine transformation, $g_{j}(.) \rightarrow h_{j}(.+w), j=1,2$.

Since the functions $g_{j} \in H(\widetilde{D})$ we can ensure that the functions $h_{j}$ still have the desired properties on $D$.

Let $\delta>0$ be so small that if we set $p:=\xi-r \nu_{\xi}$, where $0<r<\delta$ is arbitrary, we get that

$$
h_{j}(z):=g_{j}\left(z+r \nu_{\xi}\right), \quad j=1,2,
$$

will satisfy that $h_{j} \in A(D), \mathcal{Z}_{h_{1}} \cap \mathcal{Z}_{h_{2}} \cap D=\{p\}$ and furthermore that $\mathcal{Z}_{h_{1}} \cap b D \Subset V$.

Now we cover the domain with three open sets. Let $\varepsilon_{0}>0$ be so small that the ball

$$
U_{0}:=B\left(p, 2 \varepsilon_{0}\right) \Subset \omega_{r} \cap D .
$$

Choose $\varepsilon_{1}>0$ so that $\mathcal{Z}_{h_{2}} \cap U_{2}=\varnothing$, where

$$
U_{2}=\left(\left\{z \in \mathbb{C}^{2}: d_{e}\left(z, \mathcal{Z}_{h_{1}}\right)<2 \varepsilon_{1}\right\} \backslash \overline{B\left(p, \varepsilon_{0}\right)}\right) \cap \widetilde{D}_{r},
$$

where $\widetilde{D}$ is the domain from the hypothesis. If necessary, shrink $\varepsilon_{1}$ so that $U_{2} \cap b D \Subset$ $V$. Let

$$
U_{1}=\left(\left\{z \in \mathbb{C}^{2}: d_{e}\left(z, \mathcal{Z}_{h_{1}}\right)>\varepsilon_{1}\right\} \backslash \overline{B\left(p, \varepsilon_{0}\right)}\right) \cap \widetilde{D}_{r} .
$$

Obviously we have $D \Subset U_{0} \cup U_{1} \cup U_{2}$. 
Let $\left\{\phi_{j}\right\}_{j=0}^{2}$ be a partition of unity subordinate to the covering $U_{0} \cup U_{1} \cup U_{2}$ of $D$. This construction gives that $\phi_{0} \equiv 1$ on $B\left(p, \varepsilon_{0}\right), \phi_{1} \equiv 1$ on the set

$$
\left(\left\{z \in \mathbb{C}^{2}: d_{e}\left(z, \mathcal{Z}_{h_{1}}\right)>2 \varepsilon_{1}\right\} \backslash \overline{B\left(p, 2 \varepsilon_{0}\right)}\right) \cap \widetilde{D}_{r}
$$

and $\phi_{2} \equiv 1$ on the set

$$
\left(\left\{z \in \mathbb{C}^{2}: d_{e}\left(z, \mathcal{Z}_{h_{1}}\right)<\varepsilon_{1}\right\} \backslash \overline{B\left(p, 2 \varepsilon_{0}\right)}\right) \cap \widetilde{D}_{r} .
$$

Given $f \in \mathcal{B}(D)$ arbitrary such that $f(p)=0$. On $U_{0}$ we have by the OkaHefers Lemma that we can write $f(z)=f_{1}^{0}(z)\left(z_{1}-p_{1}\right)+f_{2}^{0}(z)\left(z_{2}-p_{2}\right)$, where $f_{k}^{0} \in \mathcal{B}\left(D \cap U_{0}\right), k=1,2$. (One can solve the problem on a slightly bigger ball and thereby get a solution continuous up to the boundary.) By the choice of $r$ we have that $h_{j} \in H\left(\widetilde{D}_{r}\right)$ and $D \subset \subset \widetilde{D}_{r}$. Using the Oka-Hefers Lemma again we get that

$$
h_{j}(z)=h_{1}^{j}(z)\left(z_{1}-p_{1}\right)+h_{2}^{j}(z)\left(z_{2}-p_{2}\right)
$$

and $h_{k}^{j} \in A(D), j, k=1,2$. Now let

$$
f_{k}^{j}(z)=\frac{f(z) h_{k}^{j}(z)}{h_{j}(z)}
$$

Since $h_{j} \neq 0$ on $U_{j}$ we get that $f_{k}^{j} \in \mathcal{B}\left(U_{j} \cap D\right), j=1,2, k=1,2$ and

$$
f(z)=f_{1}^{j}(z)\left(z_{1}-p_{1}\right)+f_{2}^{j}(z)\left(z_{2}-p_{2}\right)
$$

on $\overline{U_{j}} \cap \bar{D}$. So $F_{1}(z)=\sum_{j=0}^{2} \phi_{j}(z) f_{1}^{j}(z)$ and $F_{2}(z)=\sum_{j=0}^{2} \phi_{j}(z) f_{2}^{j}(z)$ give a smooth solution to the problem.

The next step will be to find a function $u$ such that

$$
f_{1}(z)=F_{1}(z)+u(z)\left(z_{2}-p_{2}\right) \text { and } f_{2}(z)=F_{2}(z)-u(z)\left(z_{1}-p_{1}\right)
$$

are in $\mathcal{B}(D)$.

Define $\lambda$ as follows:

$$
\lambda= \begin{cases}\frac{-\bar{\partial} F_{1}}{z_{2}-p_{2}} & \text { when }\left\{z_{2} \neq p_{2}\right\} \cup B\left(p, \varepsilon_{0}\right), \\ \frac{\bar{\partial} F_{2}}{z_{1}-p_{1}} & \text { otherwise. }\end{cases}
$$

This makes $\lambda$ well defined since $\frac{-\bar{\partial} F_{1}}{z_{2}-p_{2}}=\frac{\bar{\partial} F_{2}}{z_{1}-p_{1}}$ on the intersection of their domains of definition. Since $\partial^{2} \equiv 0$ we have that $\lambda$ is a $\bar{\partial}$-closed $(0,1)$-form on $D$, whose coefficients are bounded on $D$.

The support of $\lambda$ is contained in $\overline{U_{i}} \cap \overline{U_{j}}, i \neq j$. Hence we have

$$
\operatorname{supp}(\lambda) \cap b D \Subset V .
$$

By the hypothesis there exists a function

$$
u \in C(\bar{D})
$$

such that $\bar{\partial} u=\lambda$. With this $u$ we in fact have that $f_{1}, f_{2} \in \mathcal{B}(D)$. Also

$$
f(z)=f_{1}(z)\left(z_{1}-p_{1}\right)+f_{2}(z)\left(z_{1}-p_{2}\right) .
$$

So $D$ has the Gleason $\mathcal{B}$ property at $p=\xi-r \nu_{\xi}$ for $0<r<\delta$.

Since the boundary, $b D$, close to $\xi$ is $C^{1}$ smooth, we can show the same result for $p=\zeta-r \nu_{\zeta}$, where $\zeta \in b D$ is close enough to $\xi$ and $\nu_{\zeta}$ is the normal vector calculated at $\zeta$ with $0<r<\delta / 2$.

So in fact we have shown that there exists a neighborhood $O_{\xi}$ of $\xi$ in $D$ with the Gleason $\mathcal{B}$ property. 


\section{NebenhüLle AND A LOCAL $\bar{\partial}$-SOlUtion}

In this section we will show a $\bar{\partial}$-result for certain types of forms; to do this we remind the reader of the definition of the Nebenhülle. The following definition is equivalent to the one given in DF77.

Definition 1. The Nebenhülle of a domain $D$ is defined as

$$
N(D)=\text { interior }\left(\bigcap D_{\alpha}\right) \text {, }
$$

where the intersection is taken over all strictly pseudoconvex domains, $D_{\alpha}$, with smooth boundaries such that $D \Subset D_{\alpha}$.

To prove Proposition 3 we need the following proposition, which was proved in Car08b. We state the result here, since it is referred to several times in this paper.

Proposition 2 (Proposition 7, Car08b). Let $D \subset \mathbb{C}^{n}$ be a bounded pseudoconvex domain. Let $V \subset S(b D) \cap b(N(D))$ be an open set which is $C^{k}$ smooth, where $k \geq 2$.

Assume that $K$ is a nonempty, compact subset of $V$. Then there exists a bounded strictly pseudoconvex domain $\hat{D} \subset \mathbb{C}^{n}$ with $C^{k}$ regular boundary such that

(1) $D \subset \hat{D}$,

(2) $K \subset b \hat{D}$.

It is important to notice that it is only $V$ that has to have $C^{k}$ smoothness. This property is not necessary on the whole boundary $b D$. Now we are ready to state the promised $\bar{\partial}$-result.

Proposition 3. Suppose $D, K$, and $V$ satisfy the hypothesis in Proposition 2 , Assume that $f$ is a $\bar{\partial}$ closed $L_{l o c}^{2}(D)$ form such that $\sup p(f) \cap b D \subset K$. Then there exists $a(p, q-1)$ form $u$ such that $\bar{\partial} u=f$ on $D$ and such that:

(1) If $f \in L_{(p, q)}^{\infty}(D)$ and $V \in C^{2}$, then we can choose a form $u \in C_{(p, q-1)}(\bar{D})$.

(2) If $V \in C^{\infty}$ and $f \in C_{(p, q)}^{\infty}(\bar{D})$, then we can find $u \in C_{(p, q-1)}^{\infty}(\bar{D})$.

Proof. Assume $f \in L^{\infty}(D)$. Let $\hat{D}$ be the strictly pseudoconvex domain from Proposition 2. Extend $f$ trivially to $\hat{D}$. By Propositions 6.2, 6.3, and 6.4 in Øvr71 we get a solution

$$
u \in C_{(p, q-1)}(\overline{\hat{D}})
$$

and the restriction of $u$ to $\bar{D}$ is our solution to (1).

We prove (2) analogously but refer to page 229 in Koh77 instead.

Remark 2. If $\bar{D}$ is holomorphically convex, then $D$ has a Stein neighborhood basis and thereby $N(D)=D$, so $D$ satisfies the hypothesis in Proposition 2, In that case, (1) in Proposition 3 will be a special case of Theorem 1.1.b in BR80.

Remark 3. If $b D \in C^{\infty}$, then (2) in Proposition 3 is a special case of Theorem 1.1 in Bea80].

\section{The Gleason problem}

In this section we show that it is possible to solve the Gleason problem near a point $\xi \in b N(D) \cap S(b D)$ in $D$ where the boundary is $C^{2}$ smooth. Throughout this section we assume that $D$ and $V$ satisfy the hypothesis of Proposition 2 .

First we show that we can find a special type of peaking function. 
Lemma 2. For every point $\xi \in V$ there exists a function $\kappa_{\xi} \in H(\widetilde{D})$ such that $\kappa_{\xi}(\xi)=1$ and such that $\left|\kappa_{\xi}(z)\right|<1$ if $z \in \bar{D} \backslash\{\xi\}$, where $D \Subset \widetilde{D}$ and $\widetilde{D}$ is strictly pseudoconvex with a smooth boundary.

Proof. From Proposition 2 we choose $\hat{D}$ strictly pseudoconvex such that $D \subset \hat{D}$ and $\xi \in b \hat{D}, b \hat{D} \in C^{k}$.

With this it follows that there exists a function $\kappa_{\xi} \in H(\overline{\hat{D}})$ with the desired properties. (See for example Theorem VI.1.13, page 222 in Ran86.)

Since $\hat{D}$ has a Stein neighborhood basis we may assume that $\kappa_{\xi} \in H(\widetilde{D})$, where $D \subset \hat{D} \Subset \widetilde{D}$ and $\widetilde{D}$ is strictly pseudoconvex with smooth boundary.

Remark 4. Every $\xi \in V$ is a peak point to $A(D)$.

In the following, remember that $\mathcal{B} \in\left\{H^{\infty}, A\right\}$.

Proposition 4. Let $D \subset \mathbb{C}^{2}$ be a bounded pseudoconvex domain and let $V$ satisfy the hypothesis of Proposition 2, For every $\xi \in V$ there is a neighborhood $O_{\xi}$ of $\xi$ such that $D$ has the Gleason $\mathcal{B}$ property at $p$ when $p \in O_{\xi} \cap D$.

Proof. This follows from Lemma 2, Proposition 3, and Proposition 1 .

We denote the extremal points of the convex hull of $D$ as $E(\operatorname{conv}(D))$.

Corollary 1. If $D \subset \mathbb{C}^{2}$ is a bounded domain and if there exists a nonempty open set

$$
V \subset b D \cap E(\operatorname{conv}(D))
$$

such that $V \in C^{2}$, then one can solve the Gleason $\mathcal{B}$ problem close to $V$.

Proof. The convex hull has trivial Nebenhülle.

Theorem 1. Let $D \subset \mathbb{C}^{2}$ be a bounded domain such that $b D \in C^{2}$. If $\mathcal{B} \in$ $\left\{H^{\infty}, A\right\}$, then there always exist points in $D$ where we can solve the Gleason $\mathcal{B}$ problem.

Proof. There will be points of the boundary that coincide with the extremal points of its convex hull and these are of smoothness $C^{2}$.

\section{REFERENCES}

[Bea80] Frank Beatrous, Jr. Hölder estimates for the $\bar{\partial}$ equation with a support condition. Pacific J. Math., 90(2):249-257, 1980. MR600628 (82b:32029)

[BF91] U. Backlund and A. Fällström. A pseudoconvex domain with nonschlicht $H^{\infty}$-envelope. In Geometrical and algebraical aspects in several complex variables (Cetraro, 1989), volume 8 of Sem. Conf., pages 13-18. EditEl, Rende, 1991. MR.1222202 (94f:32034)

[BF95] Ulf Backlund and Anders Fällström. The Gleason problem for $A(\Omega)$. New Zealand $J$. Math., 24(1):17-22, 1995. MR.1348049 (96f:46099)

[BR80] F. Beatrous, Jr., and R. Michael Range. On holomorphic approximation in weakly pseudoconvex domains. Pacific J. Math., 89(2):249-255, 1980. MR.599118(82h:32022)

[Car08a] Linus Carlsson. Analytic properties in the spectrum of certain Banach algebras. Math. Z., 261(1):189-200, 2009. MR2452644

[Car08b] Linus Carlsson. Trivial generators for nontrivial fibres. Math. Bohem., 133(2):121-131, 2008. MR 2428308

[CCF07] Linus Carlsson, Urban Cegrell, and Anders Fällström. Spectrum of certain Banach algebras and $\bar{\partial}$-problems. Ann. Polon. Math., 90(1):51-58, 2007. MR2283112 (2008d:32009)

[Ceg93] Urban Cegrell. On the spectrum of $A(\Omega)$ and $H^{\infty}(\Omega)$. Ann. Polon. Math., 58(2):193199, 1993. MR 1239023 (94f:32031) 
[DF77] Klas Diederich and John Erik Fornaess. Pseudoconvex domains: an example with nontrivial Nebenhülle. Math. Ann., 225(3):275-292, 1977. MR0430315 (55:3320)

[FØ83] J. E. Fornæss and N. Øvrelid. Finitely generated ideals in $A(\Omega)$. Ann. Inst. Fourier (Grenoble), 33(2):v, 77-85, 1983. MR699487 (84h:32019)

[Gle64] Andrew M. Gleason. Finitely generated ideals in Banach algebras. J. Math. Mech., 13:125-132, 1964. MR0159241 (28:2458)

[Hen71] G. M. Henkin. The approximation of functions in pseudo-convex domains and a theorem of Z. L. Leǐbenzon. Bull. Acad. Polon. Sci. Sér. Sci. Math. Astronom. Phys., 19:37-42, 1971. MR0287027 (44:4234)

[Jak84] Piotr Jakóbczak. On Fornæss' imbedding theorem. Univ. Iagel. Acta Math., 24:273-294, 1984. MR815913 (87f:32038)

[Koh77] J. J. Kohn. Methods of partial differential equations in complex analysis. In Several complex variables (Proc. Sympos. Pure Math., Vol. XXX, Part 1, Williams Coll., Williamstown, Mass., 1975), pages 215-237. Amer. Math. Soc., Providence, RI, 1977. MR0477156 (57:16699)

[LW02] O. Lemmers and J. Wiegerinck. Solving the Gleason problem on linearly convex domains. Math. Z., 240(4):823-834, 2002. MR.1922731 (2003h:32008)

[OA87] Joaquín Ma and Ortega Aramburu. On Gleason's decomposition for $A^{\infty}(\bar{D})$. Math. Z., 194(4):565-571, 1987. MR881710 (88f:32053)

[Øvr71] Nils Øvrelid. Integral representation formulas and $L^{p}$-estimates for the $\bar{\partial}$-equation. Math. Scand., 29:137-160, 1971. MR0324073 (48:2425)

[Ran86] R. Michael Range. Holomorphic functions and integral representations in several complex variables, volume 108 of Graduate Texts in Mathematics. Springer-Verlag, New York, 1986. MR847923 (87i:32001)

Department of Mathematics and Mathematical Statistics, Umeå University, S-901 87 UMEÅ, SwEden

E-mail address: linus.carlsson@math.umu.se 